\title{
EFICIENCIA EN EL USO DE LOS RECURSOS Y PRODUCCIÓN MÁS LIMPIA (RECP) PARA LA COMPETITIVIDAD DEL SECTOR HOTELERO
}

\author{
Martha García Samper \\ Magister en Administración \\ Universidad de la Costa CUC \\ Barranquilla, Atlántico, Colombia \\ Mgarcia20@cuc.edu.co \\ Jesús García Guiliany \\ Doctor en Ciencias Gerenciales \\ Universidad Simón Bolivar \\ Barranquilla, Atlántico, Colombia \\ jesus.garcia@unisimonbolivar.edu.co \\ Juan Cabello Eras \\ Doutor em Ciências Técnicas \\ Universidad De la Costa \\ Barranquilla, Atlántico, Colômbia \\ jcabello2@cuc.edu.co
}

\section{RESUMEN}

El sector hotelero en el contexto Latinoamericano se enfrenta al reto de ser competitivo y sostenible. La eficiencia en el uso de los recursos y Producción más limpia (RECP) como la estrategia que integra la eficiencia productiva, protección ambiental y desarrollo humano, permitiendo crear valor y mejorar el desempeño competitivo. Este estudio caracteriza el aporte existente de la RECP y describe su potencial contribución a la competitividad en el sector hotelero del Departamento del Atlántico-Colombia, desde la perspectiva de la estrategia competitiva y la creación de valor compartido, desarrollando una investigación descriptiva con enfoque cuantitativo y análisis descriptivo en 36 hoteles mediante un instrumento con preguntas cerradas de escala nominal y Likert. Observándose que la aplicación de RECP permite obtener condiciones de efectividad económica, ambiental y social en un nivel medio, orientadas estrictamente a la maximización del beneficio económico y al cumplimiento de la regulación. Se concluye que la RECP ofrece la oportunidad de replantear las estrategias competitivas orientándolas a crear valor compartido mediante la vinculación de variables ambientales y sociales a la gestión organizacional como fuente de innovación, productividad y ventaja competitiva.

Palabras clave: Eficiencia de recursos y producción más limpia (RECP); competitividad del sector hotelero; creación de valor compartido; Departamento del Atlántico-Colombia.

\section{RESOURCE EFFICIENT \& CLEANER PRODUCTION (RECP) FOR THE COMPETITIVENESS OF THE HOSPITALITY SECTOR}

\begin{abstract}
In the Latin-American context, there is a challenge related to hotel sector, which needs to be competitive and sustainable. The Resource Efficient and Cleaner Production (RECP) as a strategy that involves productive efficiency, environmental protection, and human development, allows creating value and improving the competitive performance. From a perspective of competitive strategy and creating shared value, this study characterizes the relevance of cleaner production and describes its potential contribution to the competitiveness of the hotel sector in Atlántico, Colombia. We developed a descriptive research with a quantitative approach and a descriptive analysis in 36 hotels, through a survey with closed questions with nominal and Likert scale. The RECP allowed obtaining an economic, environmental and social effectiveness in a medium level, to maximize the profits and to fulfill the statutes. We concluded RECP gives the opportunity to rethink the competitive strategies to the creation of shared value through linking environmental and social variables with the organizational management as a source of innovation, productivity, and competitive advantage.
\end{abstract}

Keywords: Resource Efficient and Cleaner Production; competitiveness of hospitality sector; creating Shared value; Departamento Del Atlántico-Colombia. 


\section{INTRODUCCIÓN}

El turismo es uno de los sectores de mayor auge e impacto económico y ha registrado un crecimiento ininterrumpido en los últimos 10 años estimándose un aumento en las visitas de turistas internacionales del 3,3\% anual al 2030 (World Tourism Organization (UNWTO), 2016). El impacto del sector se extiende a los aspectos sociales, culturales y ambientales (Mason, 2003), entre sus componentes la hotelería genera aproximadamente el $20 \%$ de las emisiones debido a su elevado consumo de energía, agua y otros recursos (Shen \& Hsieh, 2011; World Tourism Organization (UNWTO); United Nations Environment Programme, 2008).

El medio ambiente afecta sensiblemente el desempeño organizacional (Molina et. al 2009) y para controlar el impacto organizacional sobre él se aplican diversas estrategias basadas en el control y la prevención de la contaminación (Alvarez, Burgos, \& Cespedes, 2001). La evidencia empírica confirma que las técnicas preventivas generan beneficios para los entes económicos y el medio ambiente (Molina et al 2015) y que el potencial de implementación de la eficiencia en el uso de los recursos y producción más limpia (RECP) en el sector hotelero, se orienta la eficiencia en el uso de la energía, el agua, clasificación de residuos y reducción de emisiones y vertimientos; constituyéndose en la herramienta para disminuir los impactos ambientales, reducir costos, mejorar el desempeño competitivo, la imagen corporativa y la relación con las parte interesadas (Assenova et. al 2016; Wahala et.al, 2012; United Nations Industrial Development Organization \& United Nations Environment Programme, 2010).

En Colombia, la definición de la política de producción más limpia (PML) en al año 2003 constituyó un avance en materia de sostenibilidad (Van Hoof \& Herrera, 2007). Un estudio realizado por la Alcaldía Mayor De Bogotá (2004) en instalaciones hoteleras reveló que el sector tiene el potencial para implementar la PML, reducir el consumo de recursos de todo tipo, la generación de residuos sólidos, las emisiones y los vertimientos. La implementación de la PML en Colombia ha dado como resultado la disminución del consumo de agua, energía y residuos a pesar del bajo nivel de penetración y de desarrollo de innovaciones (Van Hoof, Monroy, \& Saer, 2008).

La aplicación de la RECP, requiere migrar de un enfoque operativo a una orientación, de negocios, (Van Hoof \& Herrera, 2007), bajo la cual las iniciativas de RECP diseñadas e implementadas al margen de la estrategia competitiva, se integren y orienten a la generación de beneficios económicos, sociales, medio ambientales y a la creación de valor para todas las partes interesadas y no tan solo una visión aislada de la gestión ambiental y la sostenibilidad frente al desempeño competitivo (Porter \& Krame, 2011); partiendo del hecho de que el medio ambiente y la problemática ambiental son factores que impactan directa e indirectamente el desempeño organizacional, afectando la estrategia corporativa de los establecimientos hoteleros con alto potencial para generar ventaja competitiva (Âlvarez et.al, 2001; Molina et.al., 2009).

El presente trabajo se enfoca en realizar un diagnóstico que permita identificar los aportes alcanzados con la implementación de la RECP en el sector hotelero del Departamento Atlántico y el potencial que tiene para incrementar la competitividad desde la perspectiva sistémica de creación de valor compartido y generación de ventajas competitivas sostenibles.

\section{EFICIENCIA EN EL USO DE LOS RECURSOS Y PRODUCCIÓN MÁS LIMPIA (RECP): ALCANCE Y ÁREAS DE INTERVENCIÓN EN EL SECTOR HOTELERO.}

Un número creciente de investigaciones empíricas se han orientado a medir el impacto que la inclusión de prácticas medio ambientales en la cadena de valor o suministro ejerce sobre el desempeño sostenible de empresas de servicios como los hoteleros, concluyéndo que las iniciativas ambientales son componentes propios de la cadena de valor del servicio (Schlesinger, 1997) y que su incidencia en el desempeño de organizaciones hoteleras esta explicado mediante una mayor satisfacción y lealtad del cliente, reducción de costos, ahorro de recursos y desarrollo personal de los empleados. No obstante dada la concurrencia de una serie de factores inherentes a la naturaleza 
de los servicios hoteleros y su dependencia del entorno, las opciones para incorporar estrategias y prácticas ambientales se restringen a la prevención de la contaminación (Kassinis \& Soteriou, 2003), siendo la eficiencia en el uso de los recursos y producción más limpia, una estrategia de carácter preventivo que permite incorporar la variable ambiental en las operaciones y flujo de procesos,generando mejoras en el desempeño ambiental, social, económico y competitivo (United Nations Industrial Development Organization [UNIDO]; United Nations Environment Programme [UNEP], 2009; Li y Hamblin, 2016; Luken, et.al, 2015 y Cardoso et. al, 2016).

La investigaciones realizadas por Glavic y Lukman (2007) y Severo et al.(2015) proporcionan a partir de la revisión de la literatura el concepto de producción más limpia retomado en el año 2009 bajo el termino de eficiencia en el uso de los recursos y producción más limpia (United Nations Industrial Development Organization [UNIDO]; United Nations Environment Programme [UNEP], 2009), como una de las estrategias que posibilita la inserción de la gestión ambiental en el contexto de las organizaciones bajo una perspectiva sistémica de producción, aplicable a procesos, productos y servicios de cualquier tipo de industria, que le permite a las organizaciones, hacer uso eficiente de insumos y energía en el desarrollo de los procesos, generando un aumento de la productividad, competitividad y el mejoramiento del rendimiento organizacional.

-La implementación de la eficiencia en el uso de los recursos y producción más limpia, impacta los aspectos tecnológicos, administrativos y culturales en las organizaciones y las comunidades donde estas se sitúan (Van Berkel, 2015), identificándose su presencia en las funciones de planificación y organización al propiciar la implementación de sistemas de gestión ambiental que proporciona las bases para administrar los aspectos ambientales de manera coherente y sostenida; en las operaciones, las cuales apuntan al cambio o mejoramiento tanto en el diseño como en el desarrollo de las actividades, involucrando a los diferentes agentes, manteniendo una visión a largo plazo, o bien comunicando las iniciativas y los avances en el desempeño ante los grupos de interés como parte de su compromiso social-ambiental y el interés de construir una imagen corporativa sólida y favorable (Gonzalez \& Gonzalez, 2005).

Situación que se materializa a través de la implementación de buenas prácticas orientadas a racionalizar el consumo de recursos, reutilizar los insumos y reemplazar materias primas tóxicas, modificación de métodos de trabajo, rediseño de servicios, incorporación de tecnologías más eficientes y menos nocivas para el medio ambiente, adopción de sistemas de gestión ambiental y el cambio de los modelos de pensamiento con relación a la gestión de los aspectos ambientales en todos los niveles y procesos de la organización, impactando el desempeño y respondiendo a la demanda de servicios verdes (Lin et. al, 2013; Van Berkel, 2015; Cardoso et.al. 2016), dentro de los cuales se incluyen las fases de construcción, operación, prestación y disfrute del servicio (Carbo, 2013), presentadas en figura 1. 
Figura 1. RECP en la cadena de valor del servicio del sector hotelero

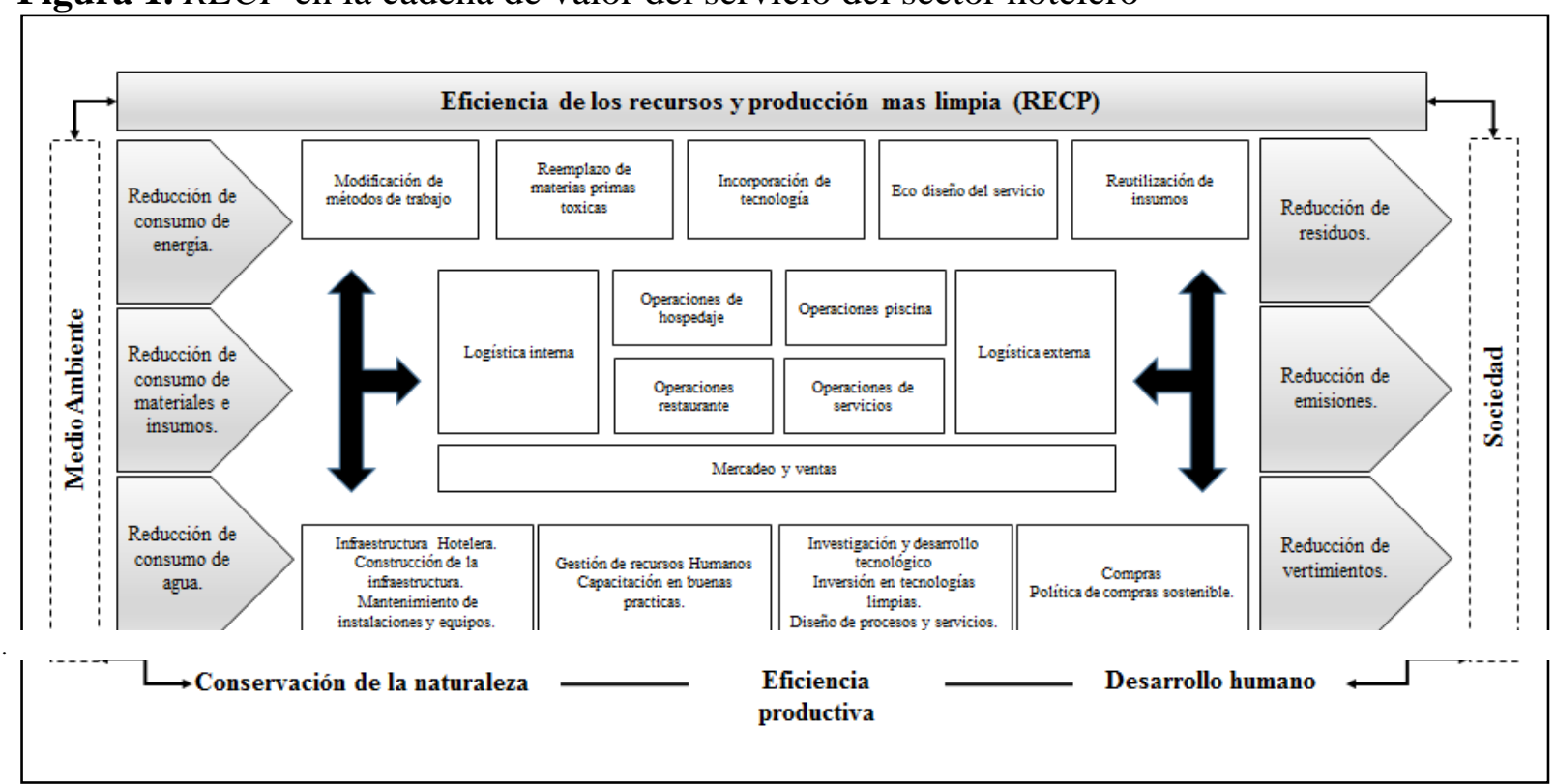

Fuente: Elaboración propia (2016) con base en United Nations Industrial Development Organization [UNIDO]; United Nations Environment Programme [UNEP], (2009); Alcaldía Mayor De Bogotà;Unidad de Asistencia Técnica y ambiental para la Pequeña y mediana empresa [ACERCAR], Banco Interamericano de Desarrollo [BID]; Asociacion Colombiana de las micro, pequeñas y medianas empresas [ACOPI]; Corporación para la investigación socioeconomica y tecnológica de Colombia [CINCET],(2004).

En la representación, se observa la inserción de la $R E C P$ en la cadena de valor del sector hotelero, interviniendo desde la concepción de su infraestructura y en el desarrollo de los procesos críticos a través de la aplicación de prácticas, programas, inversiones e innovaciones que permiten incorporar a la gestión de la organización la "eficiencia productiva", "conservación de la naturaleza y "desarrollo humano" como ejes transversales capaces de contribuir con el desempeño económico ambiental y social de la organización.

\section{APORTES DEL USO EFICIENTE DE RECP A LA COMPETITIVIDAD DEL SECTOR HOTELERO}

La variable ambiental es un factor de gran importancia para la sostenibilidad y desempeño competitivo del sector hotelero (Molina et al.,2009; Amado dos Santos, et al., 2017); constituyéndose la RECP en la estrategia que permite gestionar, de manera independiente y conjunta, la "eficiencia productiva", "conservación del medio ambiente" y "desarrollo humano" (United Nations Environment Program [UNEP], 2016), permitiendo que empresas, empleados, medio ambiente, clientes y sociedad sean beneficiados (Dodic et.al.,2010), con la optimización del desempeño organizacional, diseño de servicios ambientalmente sostenibles mejora de las condiciones de salud y calidad de vida de empleados y comunidad, así como las condiciones medioambientales, armonizando la relación entre economía y medio ambiente, y colocando las estrategias ecológicas al servicio de los sistemas productivos (Kjaerheim \& Askham, 2005, Van Hoof, Monroy y Saer, 2007, Cabello et.al. 2015, Severo et al., 2015; Luken et al 2015).

Así entonces, el documento elaborado por la Organización de las Naciones Unidas para el Medio Ambiente [PNUMA] (2003), da a conocer las bases para definir las variables del estudio, al describir los efectos generados por acciones correctivas y de RECP en la implementación de los acuerdos ambientales multilaterales, refiriéndose a la efectividad económica, ambiental y social como el resultado de la aplicación de la estrategia; Asimismo, Henriques \& Catarino (2015), mediante estudios realizados en pequeñas y medianas empresas, concluye que la aplicación de la metodología de valor sostenible teniendo como estrategia de apoyo la PML, genera resultados de 
carácter económico, ambiental y social tales como, la reducción y control de costos, incremento de las utilidades a través del uso eficiente de los recursos, reducción del consumo de recursos, implementación de mejores prácticas ambientales, adecuada comunicación y respuesta a las necesidades de sus principales grupos de interés y desarrollo de competencias y actitudes ambientalmente sostenibles en el trabajo.

La efectividad económica es el resultado de la aplicación de la PML, en términos de generación de ingresos económicos, reducción de costos y oportunidades para la innovación (Organización de las Naciones Unidas para el Medio Ambiente [PNUMA] 2003); asimismo Molina et al., (2009b) en estudio realizado para medir el impacto de las prácticas ambientales en el desempeño organizacional, concluye que variables como el "desempeño competitivo" relacionada con estrategias de diferenciación y reducción de costos, aumentan con el grado de proactividad ambiental del hotel, dimensión expresada mediante la reducción del consumo de productos peligrosos, prácticas de ahorro de agua y energía, cuantificación de ahorros y costos, entre otros; al respecto de esta última variable, Cabello et al., (2016) resalta la importancia de definir indicadores de desempeño energético, como medio para identificar malas prácticas y desperdicios.

Por su parte, Zeng et.al. (2010), tras estudiar el impacto de la producción más limpia en el desempeño de los negocios concluye que las actividades realizadas bajo un esquema de alto costo en el marco de la implementación de producción más limpia, representadas por inversiones tecnológicas, innovación en los procesos y productos, adquisición de recursos renovables e investigación para la protección ambiental, contribuyen significativamente al desempeño financiero, mientras que las de un esquema de bajo costo, orientadas a implementar buenas prácticas, mejorar ambientes de trabajo, incrementar el reciclaje y concientizar a las personas de la importancia de la $R E C P$, realizan un gran aporte al desempeño no financiero, por ejemplo a la imagen corporativa.

La implementación de la RECP, permite aumentar los niveles de innovación al actuar como herramienta para estudiar el ciclo de vida del producto y emprender acciones de diseño ecológico que permitan reducir el impacto ambiental (Severo et al., 2015), asi como la implementación de innovaciones tecnologicas que segun Severo et al., (2012) y Zeng et.al. (2010), incrementan la eficiencia operacional, reducen los costos y posibilitan la producción de servicios de calidad ambiental pertinentes a la demanda del mercado.

La efectividad ambiental según la Organización de las Naciones Unidas para el Medio Ambiente [PNUMA] (2003), está relacionada con la reducción de los impactos ambientales y el aumento de la eficiencia en el uso de los recursos, asimismo, Severo et al, (2012,2015), la define como la inclusión de la variable ambiental en los procesos productivos y en el ciclo de vida del servicio, que posibilita la reducción de residuos e impactos perjudiciales sobre el medio ambiente, confirmando mediante estudio empírico que tenía como objetivo analizar la relación entre la producción más limpia, sostenibilidad y desempeño organizacional, la positiva relación de estas prácticas en la sostenibilidad ambiental a través de la optimización del consumo de recursos naturales y materiales que son utilizados como insumos de entrada.

En este sentido de acuerdo a lo indicado por Oreja \& Armas (2012), dada la naturaleza del servicio en el sector hotelero los impactos son generados en el establecimiento mismo, como resultado del consumo de recursos y emisiones asociadas a la producción y disfrute simultaneo del servicio de alojamiento, lo cual hace necesario que se establezcan estrategias de proactividad ambiental que involucren al cliente a través del aprendizaje y aplicación de comportamientos amigables con el medio ambiente.

El potencial de la RECP de crear valor social para todas las partes interesadas y lograr la participación de los diferentes actores en la estrategia (Organización de las Naciones Unidas para el Medio Ambiente [PNUMA] 2003), es constatado por estudios realizados en diferentes contextos empresariales y geográficos, Severo et al, (2015) lo confirma al medir la contribución de la PML al desarrollo de la salud y seguridad en el trabajo y encontrar que en las organizaciones metalmecánicas brasileras se genera este aporte; desde el concepto expresado por Dodic et.al.(2010), la PML es uno de los instrumentos más eficaces para disminuir el riesgo que los 
materiales tóxicos representan para los seres humanos generando una serie de beneficios para la sociedad y los empleados a partir de la disminución de la contaminación, prevención de accidentes y compromiso con el talento humano, de igual forma, el análisis bibliométrico realizado por Amado dos Santos, et.al. (2017) en el contexto del sector hotelero, resalta la necesidad de que se definan criterios integrales de gestión que posibiliten la protección del medio ambiente, la cultura y las adecuadas condiciones económicas y políticas para la sostenibilidad de la actividad hotelera.

Teniendo como base los estudios considerados en la revisión de la literatura, se sintetizan los aportes de la RECP a la competitividad en tabla 2.

\begin{tabular}{|c|c|c|c|}
\hline Autor & Beneficios Económicos & Beneficios ambientales & Beneficios Sociales \\
\hline $\begin{array}{l}\text { Organización de las } \\
\text { Naciones Unidas } \\
\text { para el Medio } \\
\text { Ambiente [PNUMA] } \\
(\mathbf{2 0 0 3 )}\end{array}$ & $\begin{array}{l}\text {-Ganancias de } \begin{array}{r}\text { económicas- } \\
\text { Desarrollo } \\
\text { innovadoras }\end{array} \\
\text {-Reducción de costos }\end{array}$ & $\begin{array}{l}\text {-Mejora la eficiencia en el uso } \\
\text { de } \\
\text { Recursos. } \\
\text {-Reducir los impactos } \\
\text { ambientales }\end{array}$ & $\begin{array}{l}\text { Motivar el dialogo entre } \\
\text { actores } \\
\text { Incentivos para los actores } \\
\text { de la implementación. }\end{array}$ \\
\hline Zeng et.al.(2010) & $\begin{array}{l}\text {-Decrecimiento de costos de } \\
\text { producción. } \\
\text {-Desempeño Financiero } \\
\text {-Desempeño no financiero }\end{array}$ & & \\
\hline Molina et al., (2009) & $\begin{array}{l}\text {-Prácticas de ahorro de energía y } \\
\text { agua }\end{array}$ & $\begin{array}{ll}\text {-Reducción } & \text { del uso de } \\
\text { productos } & \text { ambientalmente } \\
\text { peligrosos } & \end{array}$ & $\begin{array}{l}\text {-Satisfacción de los grupos } \\
\text { de interés (empleados y } \\
\text { clientes). }\end{array}$ \\
\hline $\begin{array}{l}\text { Severo et al, } \\
(2012,2015)\end{array}$ & $\begin{array}{l}\text {-Reducción de costos de } \\
\text { producción } \\
\text {-Incremento de la eficiencia y la } \\
\text { productividad } \\
\text {-Desarrollo de innovaciones } \\
\text { tecnológicas } \\
\text {-Desarrollo de innovaciones en } \\
\text { el producto } \\
\text {-Reducción de pasivos } \\
\text { ambientales }\end{array}$ & $\begin{array}{l}\text {-Reducción del consumo de } \\
\text { agua, energía y materiales } \\
\text { peligrosso } \\
\text {-Reutilización de recursos } \\
\text {-Incremento de eficiencia } \\
\text { ecológica. } \\
\text {-Contribuye a reducir el } \\
\text { impacto en el medio ambiente }\end{array}$ & $\begin{array}{l}\text {-Incremento del nivel de } \\
\text { satisfacción de los clientes. } \\
\text {-Desarrollo de condiciones } \\
\text { de seguridad y } \\
\text { salud de los colaboradores. }\end{array}$ \\
\hline Dodic et.al.(2010) & $\begin{array}{l}\text {-Reducción en costos de } \\
\text { tratamiento y disposición de } \\
\text { desechos de materias primas } \\
\text { y } \\
\text {-Desarrollo de productos } \\
\text { ecológicos. }\end{array}$ & $\begin{array}{l}\text {-Disminución del consumo de } \\
\text { recursos } \\
\text { contaminación residuos } \\
\text { peligrosos. y efectos negativos } \\
\text { en el medio ambiente. }\end{array}$ & $\begin{array}{l}\text {-Prevención de accidentes } \\
\text {-Mayor compromiso con el } \\
\text { talentororán de la salud } \\
\text {-Preservación dumano. } \\
\text { de los habitantes. }\end{array}$ \\
\hline $\begin{array}{c}\text { Henriques y } \\
\text { Catarino (2015) }\end{array}$ & $\begin{array}{l}\text {-Diagnóstico y mejora de los } \\
\text { procesos. } \\
\text {-Identificación, control y } \\
\text { reducción de costos. } \\
\text {-Reducción de materiales, } \\
\text { energía y agua. } \\
\text {-Aumento de la rentabilidad. }\end{array}$ & $\begin{array}{l}\text {-Implementación de mejores } \\
\text { prácticas ambientales. } \\
\text {-Reducción del consumo de } \\
\text { energía y agua. } \\
\text {-Reducción de residuos y } \\
\text { emisiones. }\end{array}$ & $\begin{array}{lcr}\text {-Mejora } & \text { de } & \text { la } \\
\text { comunicación } & \text { interna } & \text { y } \\
\text { externa. } & & \\
\text {--Desarrollo } & & \text { de } \\
\text { competencias para } & \text { la } \\
\text { responsabilidad social. } & \end{array}$ \\
\hline
\end{tabular}

Tabla 1. Aportes de la RECP a la competitividad

Fuente. Elaboración propia (2016) con base en revisión de la literatura.

\section{COMPETITIVIDAD EN EL SECTOR HOTELERO BASADA EN EL MODELO ESTRATÉGICO DE LA GENERACIÓN DE VALOR ECONÓMICO A LA CREACIÓN DE VALOR COMPARTIDO}

Teniendo en cuenta los estudios de Porter, (1991) la competitividad se hace manifiesta en la capacidad de las organizaciones para generar ventajas sobre su competencia, resultante de la selección y exitosa implementación de estrategias competitivas de generación de valor; Sánchez y Cebrián (2015), lo abordan en el contexto turístico y hotelero, destacando que el destino se 
constituye en recurso, factor de ventaja comparativa y competitiva que define la estrategia y la oferta de los establecimientos, concentrando una serie de atributos sociales, culturales y paisajísticos particulares y no transferibles a otros contextos; vinculados a la generación de valor económico y compartido.

La generación de valor económico es concebido como la maximización del beneficio económico y patrimonio de los accionistas, siendo considerada como su única responsabilidad y forma de generar bienestar para la sociedad, a la cual es posible llegar a través de la aplicación de estrategias de diferenciación o liderazgo en costes que permitan por un lado aumentar los beneficios percibidos por el cliente y por otro reducir los costos de operación generando así una posición de ventaja competitiva. (Porter 1991, Fernández 2009; Freeman et al. 2014),

La responsabilidad social se define como una filosofía que surge en respuesta y contraposición a una actividad empresarial ajena a su entorno, procurando que las organizaciones asuman un compromiso voluntario y públicamente demostrable de contribuir con el bienestar social, la conservación del medio ambiente, alineado a la estrategia de negocios y que vincule a la organización con la competitividad, sin embargo ante la separación entre la empresa y la sociedad, este concepto se plantea como una redistribución del valor económico generado entre las necesidades de la sociedad, evidenciándose la ausencia de un enfoque estratégico e (Porter \& Van Der Linde 1995; Porter \& Krame 2011 a ; Porter \& Kramer 2006).

La creación de valor compartido, es definida por Porter \& Krame, (2011), como la integración de las necesidades sociales a la gestión de las organizaciones a través de un proceso cooperativo de construcción de valor apoyándose en la ejecución de estrategias de diferenciación y liderazgo en costo, derivada del repensar de las actividades de la cadena de valor y del concepto de productividad, en función de las necesidades y realidades de la población y el entorno, permitiendo que las dinámicas ambientales y sociales contribuyan al mejoramiento del desempeño organizacional y se conviertan en la base para la generación de ventaja competitiva, sostenibilidad y responsabilidad. De acuerdo a Figge y Hahn (2004), la transición hacia el mencionado modelo requiere de la identificación y cuantificación por parte de la empresa de los recursos que utiliza y en los cuales incide durante el ejercicio de sus actividades, para determinar su capacidad de generar valor compartido sostenible con una imagen corporativa que la diferencie en el mercado de acción.

Serra \& Font, (2016) señalan que la creación de valor compartido tiene como base el desarrollo de una gestión responsable de las operaciones en su relación con los diferentes factores del entorno con el mínimo conflicto de intereses, propiciando condiciones de solidez en la industria, mejoramiento de la calidad de vida de las poblaciones y conservación del medio ambiente consolidándose como una estrategia para potenciar el desarrollo competitivo de la hotelería sostenible al conciliar las variables ambientales y sociales, a través de una gestión fundamentada en los principios económico-estratégicos, medioambientales, sociales y culturales (Instituto de sostenibilidad turística, 2011).

El propósito de las organizaciones hoteleras de trabajar bajo un paradigma de creación de valor compartido, encuentra en las estrategias de gestión ambiental preventiva como la $R E C P$, condiciones propicias para generar beneficios en las tres dimensiones del desarrollo sostenible (United Nations Industrial Development Organization [UNIDO]; United Nations Environment Programme [UNEP], 2009), como consecuencia de procesos de innovación, mejoramiento continuo, eficiencia en el uso de los recursos, y desarrollo de procesos que permiten reducir costos, diferenciar los servicios (Molina et al., 2009) y mejorar las condiciones de seguridad y calidad de vida de clientes internos y sociedad en general (Dodic et.al,2010; Severo et al, 2015).

\section{ENFOQUE METODOLÓGICO}

La investigación se realizó mediante un abordaje descriptivo, de campo, con enfoque cuantitativo; aspectos metodológicos que permiten cumplir el objetivo de caracterizar los aportes reales y potenciales de la RECP a la competitividad del sector Hotelero del Departamento del 
Atlántico. Como la investigación descriptiva, se orienta a exponer la situación estudiada en función de sus principales características, el diseño de campo es utilizado en casos donde el fenómeno es estudiado directamente, en tiempo y espacio real (Hurtado, 2010) y el enfoque cuantitativo permite medir las características de los fenómenos y realizar generalizaciones en cuanto a los resultados (Bernal, 2010) mediante técnicas estadísticas (Hernández, Fernández, \& Baptista, 2010).

Para la recolección de los datos se utilizó la técnica de la encuesta aplicando como instrumento un cuestionario con escalas de medición de tipo nominal, que fue validado a través de la medida de consistencia interna "coeficiente alfa de Cronbach", presentando un índice de confiabilidad del 0,882, que de acuerdo a los parámetros de interpretación proporcionados por Hernández et.al (2010) se situa como un grado elevado de confiabilidad. Para el diseño del cuestionario se tuvieron en cuenta, las dimensiones e indicadores obtenidos a partir de los referentes teóricos y la revisión de la literatura, lo cual permitió validar el constructo teniendo en cuenta la forma como la variable ha sido medida en otras investigaciones. En la tabla siguiente se muestra el sistema de variables, escala de medición y componentes de la revisión de la literatura considerados en el diseño del cuestionario.

\begin{tabular}{|c|c|c|c|c|}
\hline Dimensión & Subdimensión & Indicador & Escala de Medición & $\begin{array}{l}\text { Revisión de la } \\
\text { literatura }\end{array}$ \\
\hline \multirow{8}{*}{ 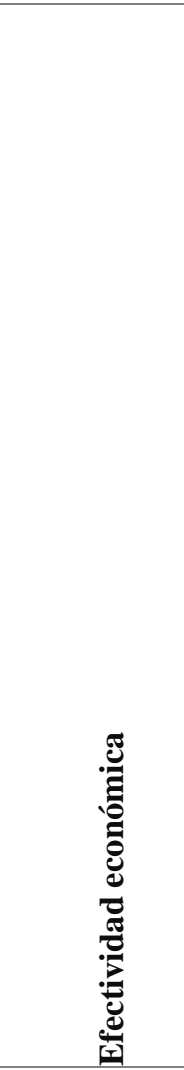 } & \multirow{3}{*}{$\begin{array}{l}\text { Incremento de las } \\
\text { utilidades }\end{array}$} & $\begin{array}{l}\text { Actividades de alta y } \\
\text { baja inversión }\end{array}$ & Nominal & \multirow{8}{*}{$\begin{array}{l}\text { Organización } \\
\text { de las } \\
\text { Naciones } \\
\text { Unidas para el } \\
\text { Medio } \\
\text { Ambiente } \\
\text { [PNUMA] } \\
\text { (2003); Zeng } \\
\text { et.al.(2010); } \\
\text { Molina et al., } \\
\text { (2009); Severo } \\
\text { et. al.,(2012) }\end{array}$} \\
\hline & & $\begin{array}{l}\text { Nivel de Rendimiento a } \\
\text { la inversión. }\end{array}$ & $\begin{array}{l}\text { Nominal } \\
\text { Ninguno (1), bajo (2) } \\
\text { Medio (3), alto (4) }\end{array}$ & \\
\hline & & $\begin{array}{l}\text { Horizonte de tiempo } \\
\text { del Rendimiento a la } \\
\text { Inversión }\end{array}$ & $\begin{array}{l}\text { Nominal } \\
\text { Ninguno (1), a corto plazo (2), a } \\
\text { mediano plazo ( } 3 \text { ), a largo plazo } \\
\text { (4). }\end{array}$ & \\
\hline & \multirow{3}{*}{$\begin{array}{l}\text { Reducción de } \\
\text { costos }\end{array}$} & $\begin{array}{l}\text { Reducción de costos } \\
\text { de servicios públicos }\end{array}$ & $\begin{array}{l}\text { Nominal } \\
\text { Nulo (1), bajo (2), medio ( } 3) \text {, } \\
\text { alto (4) }\end{array}$ & \\
\hline & & $\begin{array}{l}\text { Costos por compras de } \\
\text { materiales e insumos }\end{array}$ & $\begin{array}{l}\text { Nominal } \\
\text { Altos (1), estables (2), bajos ( } 3 \text { ), } \\
\text { muy bajos (4) }\end{array}$ & \\
\hline & & $\begin{array}{l}\text { Reducción de costos de } \\
\text { servicios públicos de } \\
\text { recolección }\end{array}$ & $\begin{array}{l}\text { Nominal } \\
\text { Nulo (1), bajo ( } 2) \text {, medio ( } 3) \text {, } \\
\text { alto (4) }\end{array}$ & \\
\hline & \multirow[t]{2}{*}{ Innovación } & $\begin{array}{l}\text { Nivel de innovación en } \\
\text { infraestructura del } \\
\text { servicio- } \\
\text { Programas/acciones de } \\
\text { ahorro de recursos }\end{array}$ & $\begin{array}{l}\text { Nominal } \\
\text { Nulo (1), bajo ( } 2) \text {, medio ( } 3 \text {, } \\
\text { alto (4) }\end{array}$ & \\
\hline & & $\begin{array}{l}\text { Nivel de innovación en } \\
\text { la prestación del } \\
\text { servicio- }\end{array}$ & $\begin{array}{l}\text { Nominal } \\
\text { Nulo ( } 1) \text {, bajo ( } 2) \text {, medio ( } 3) \text {, } \\
\text { alto (4) }\end{array}$ & \\
\hline
\end{tabular}




\begin{tabular}{|c|c|c|c|c|}
\hline Dimensión & Subdimensión & Indicador & Escala de Medición & $\begin{array}{l}\text { Revisión de la } \\
\text { literatura }\end{array}$ \\
\hline \multirow{6}{*}{ 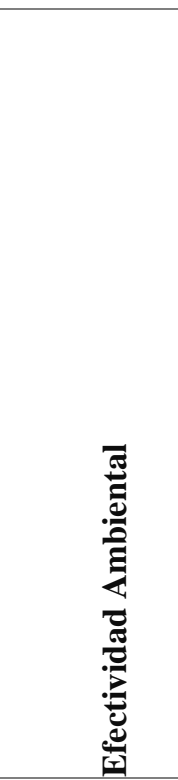 } & \multirow{3}{*}{$\begin{array}{l}\text { Aumento de la } \\
\text { eficiencia en el uso } \\
\text { de los recursos. }\end{array}$} & $\begin{array}{l}\text { Nivel de eficiencia en el } \\
\text { consumo de la energía }\end{array}$ & $\begin{array}{l}\text { Nominal } \\
\text { Bajo (1), estable (2), } \\
\text { alto (3), muy alto (4) }\end{array}$ & \multirow{6}{*}{$\begin{array}{l}\text { Organización de las } \\
\text { Naciones Unidas } \\
\text { para el Medic } \\
\text { Ambiente } \\
\text { [PNUMA] } \\
\text { (2003);Molina e } \\
\text { al., (2009), Severc } \\
\text { et al, (2012-2015) } \\
\text { Dodic } \\
\text { et.al.(2010); Henriq } \\
\text { ues y Catarino }\end{array}$} \\
\hline & & $\begin{array}{l}\text { Nivel de eficiencia en el } \\
\text { consumo del agua. }\end{array}$ & $\begin{array}{l}\text { Nominal } \\
\text { Nulo (1), bajo (2), } \\
\text { medio (3), alto (4) }\end{array}$ & \\
\hline & & $\begin{array}{l}\text { Nivel de eficiencia en el } \\
\text { consumo de materiales e } \\
\text { insumos }\end{array}$ & $\begin{array}{l}\text { Nominal } \\
\text { Nulo (1), bajo (2), } \\
\text { medio (3), alto (4) }\end{array}$ & \\
\hline & \multirow{3}{*}{$\begin{array}{l}\text { Reducción del } \\
\text { impacto ambiental } \\
\text { y la contaminación }\end{array}$} & $\begin{array}{l}\text { Impacto } \\
\text { asociado al consumo de } \\
\text { agua. }\end{array}$ & $\begin{array}{l}\text { Nominal } \\
\text { Alto (1), estable (2), } \\
\text { bajo (3), muy bajo (4) }\end{array}$ & \\
\hline & & $\begin{array}{l}\text { Aplicación de medidas } \\
\text { para reducir el consumo de } \\
\text { insumos tóxicos. }\end{array}$ & $\begin{array}{l}\text { Ordinal } \\
\text { Nunca (1), casi nunca } \\
(2) \text {, algunas veces (3), } \\
\text { siempre (4) }\end{array}$ & \\
\hline & & $\begin{array}{l}\text { Contaminación asociada a } \\
\text { la disposición de recursos }\end{array}$ & $\begin{array}{l}\text { Nominal } \\
\text { Alto (1), estable (2), } \\
\text { bajo (3), muy bajo (4) }\end{array}$ & \\
\hline \multirow{3}{*}{ 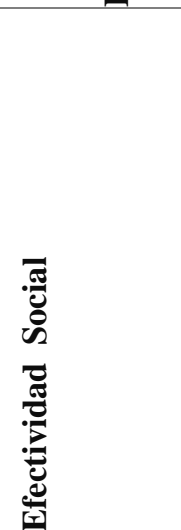 } & $\begin{array}{l}\text { Reducción de } \\
\text { riesgos laborales }\end{array}$ & $\begin{array}{l}\text { Nivel de riesgos laborales } \\
\text { tras la implementación de } \\
\text { acciones de RECP. }\end{array}$ & $\begin{array}{l}\text { Nominal } \\
\text { Alto (1), medio (2), } \\
\text { bajo (3), muy bajo (4) }\end{array}$ & \multirow{3}{*}{$\begin{array}{l}\text { Severo et al, (2012- } \\
2015) ; \quad \text { Dodic } \\
\text { et.al.(2010), } \\
\text { Henriques } \\
\text { Catarino (2015) }\end{array}$} \\
\hline & $\begin{array}{l}\text { Participación } \\
\text { activa de todas las } \\
\text { partes involucradas }\end{array}$ & $\begin{array}{l}\text { Participación de los grupos } \\
\text { de interés en la planeación } \\
\text { e implementación de } \\
\text { acciones de RECP }\end{array}$ & $\begin{array}{l}\text { Nominal } \\
\text { Muy baja (1), baja (2), } \\
\text { parcial (3), alta (4) }\end{array}$ & \\
\hline & $\begin{array}{l}\text { Calidad de vida de } \\
\text { las comunidades } \\
\text { aledañas. }\end{array}$ & $\begin{array}{l}\text { Contribución de las } \\
\text { acciones de producción } \\
\text { más limpia a la calidad de } \\
\text { vida de las comunidades } \\
\text { aledañas. }\end{array}$ & $\begin{array}{l}\text { Nominal } \\
\text { No contribuye (1), bajo } \\
(2), \text { medio (3), alta (4) }\end{array}$ & \\
\hline
\end{tabular}

En función de las escalas de medición y tipo de datos obtenidos los datos recolectados fueron interpretados y analizados mediante técnicas descriptivas, (Hurtado, 2010), representadas por la distribución de frecuencias y el cálculo de la moda como estadístico descriptivo que permite determinar la opción de respuesta más representativa entre las alternativas disponibles en cada una de las dimensiones establecidas.

El enfoque metodológico utilizado, se corresponde con los estudios realizados por Cabello et. al. (2015) en cuanto al tipo de investigación (descriptiva), la tecnica e instrumento utilizado (encuesta) para estudiar la implementacion de PMLen la ciudad de Cienfuegos y el estudio realizado por Ulzbach, Da Silva, \& Hein (2015) en cuanto a la aplicación del enfoque cuantitativo y el alcance descriptivo para analizar la relación entre responsabilidad social y el desempeño economico-financiero de organizaciones participantes en el indice de sustentabilidad empresarial

La población de estudio está representada por 40 establecimientos hoteleros medianos y grandes clasificados en función de su oferta en habitaciones, identificados con Código de Clasificación Uniforme (CIIU) 5511, Registro Nacional de Turismo activo (Ver tabla 3), seleccionados los medianos y grandes establecimientos en función del mayor nivel de participación que presentan, con respecto al resto de la población. La muestra fue calculada mediante el muestreo aleatorio estratificado, partiendo de un nivel de error del 5\%, de confianza esperado del $95 \%$ y una proporción de elementos con las características deseadas del 50\%, obteniéndose una muestra de 36 organizaciones distribuidas en 32 empresas medianas y 4 grandes. 
Oferta en habitaciones.

Criterio

Población

Ámbito

geográfico

Medianas (50 a 150 habitaciones), y grandes (mayor a 150

habitaciones),

Según la clasificación propuesta en la Norma Técnica Sectorial 5133: Etiquetas ambientales Tipo I.

Empresas con Registro Nacional de Turismo. Estado activo.

Empresas identificadas con el Código de Clasificación

Uniforme (CIIU) 5511: Alojamiento en hoteles, hostales y

aparta hoteles.

Tabla 3. Criterios de selección de hoteles en el estudio.

Fuente. Elaboración propia (2016) con base en Instituto Colombiano de Normas Técnicas [ICONTEC] y Ministerio de ambiente y desarrollo sostenible (2006), Registro Unico Empresarial y social Camaras de Comercio [RUES]- Registro Nacional de Turismo. (2015)

\section{ANÁLISIS Y DISCUSIÓN DE LOS RESULTADOS.}

En esta sección se describen y analizan los resultados obtenidos, mediante técnicas de análisis descriptivo, como las medidas de dispersión (Distribución de frecuencias) y tendencia central (moda), utilizando para su procesamiento el software de análisis estadístico SPSS.

En consideración a las cuatro categorías de respuesta definidas en escalas de medición nominal para cada uno de los indicadores y con el fin de caracterizar el grado de existencia de las dimensiones estudiadas, en tabla 4 se presenta baremo con intervalos en términos porcentuales que categoriza el nivel de condiciones de efectividad económica ambiental y social.

\begin{tabular}{cc}
\hline Categoría & Rango (\%) \\
\hline $\begin{array}{c}\text { Nivel Alto de condiciones } \\
\text { De efectividad }\end{array}$ & $76 \leq \mathrm{x} \leq 100$ \\
\hline $\begin{array}{c}\text { Nivel medio de condiciones } \\
\text { De efectividad }\end{array}$ & $51 \leq \mathrm{x}<75$ \\
\hline $\begin{array}{c}\text { Nivel bajo de condiciones } \\
\text { De efectividad }\end{array}$ & $26 \leq \mathrm{x}<50$ \\
\hline $\begin{array}{c}\text { Nivel muy bajo de condiciones } \\
\text { De efectividad }\end{array}$ & $1 \leq \mathrm{x}<25$ \\
\hline
\end{tabular}

Tabla 4. Baremo nivel de condiciones de efectividad.

Fuente. Elaboración propia (2016) con base en revisión de la literatura y diseño de la encuestas.

La tabla 10 evidencia los resultados de la valoración realizada por los gerentes con relación a los aportes económicos derivados de la implementación de prácticas e iniciativas de RECP, en las organizaciones que representan, observándose la presencia de condiciones de efectividad económica con un nivel medio en la muestra estudiada correspondiente a un $54 \%$.

\begin{tabular}{llc}
\hline \multicolumn{1}{c}{ Dimensión } & \multicolumn{1}{c}{ Indicador } & \% \\
\hline \multirow{2}{*}{ Efectividad económica. } & Incremento de las utilidades. & 50 \\
\cline { 2 - 3 } & Reducción de los costos & 69 \\
\cline { 2 - 3 } & & 42 \\
\hline & Aumento Innovación. & 54 \\
\hline
\end{tabular}


El comportamiento presentado en el indicador incremento de las utilidades comprende el conjunto de resultados económicos y financieros derivados de las inversiones en actividades de producción más limpia, representado en el caso de estudio a través de un mayor uso de actividades de PML de baja inversión ante las cuales el indicador nivel de retorno a la inversión con la tendencia de respuesta 4, evidencia un alto grado de recuperación, que ocurre en un corto, mediano y largo plazo, con la opción 3 (mediano plazo) como respuesta característica y la presencia de un porcentaje minoritario de organizaciones que manifiestan no haberlos cuantificado (ver figura 2). Los resultados presentados coinciden con los hallazgos de estudios realizados en organizaciones hoteleras e industriales, donde se demuestra que un desempeño ambiental proactivo y la aplicación de estrategias de PML contribuyen al desempeño económico y financiero en términos del margen de utilidades operacionales, retorno a la inversión y rentabilidad (Zeng et.al.2010; Molina et al., 2009).

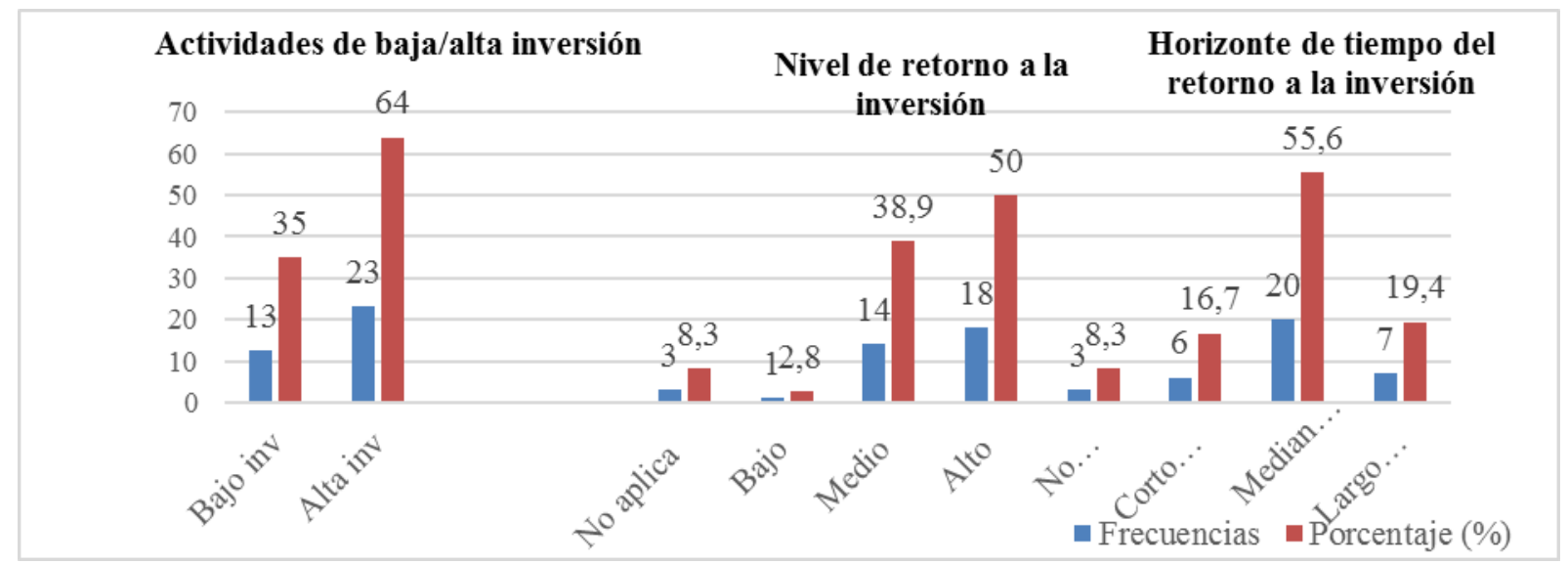

Figura 2. Resultados Indicador Incremento de utilidades.

Fuente: Elaboración propia (2016) con base en resultados de encuestas (2016)

Las organizaciones hoteleras manifiestan haber percibido reducciones en los costos mediante mayores ahorros en pagos de servicios públicos y la disminución de los costos asociados a compras de materiales e insumos, seleccionando de manera mayoritaria la opción 4 (alto), y la opción 3 (bajo) respectivamente; tendencia que se confirma en la frecuencia y porcentaje de respuesta de cada una de las opciones (Ver figura 3) y confirma en el contexto de los medianos y grandes hoteles, la validez de las afirmaciones de Severo et al (2012; 2015) y Dodic et.al.,(2010), con respecto a la contribución que hacen las metodologías de PML en el desempeño organizacional, mediante la reducción de los costos de operación y el aumento de la eficiencia en los procesos, considerados como aspectos que aumentan las posibilidades de éxito competitivo (Molina et. al, 2009).

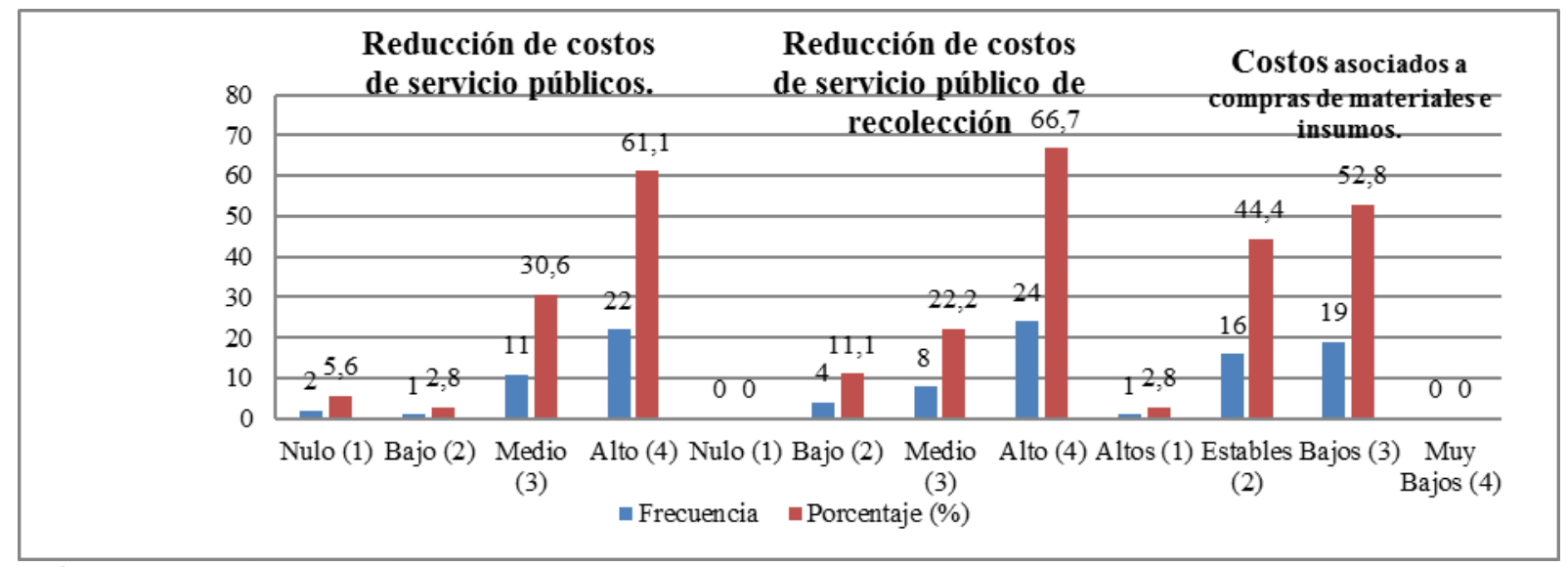

Figura 3. Resultados indicador reducción de costos.

Fuente: Elaboración propia (2016) con base en resultados de encuestas (2016)

Revista de Gestão Social e Ambiental - RGSA, São Paulo, v. 11, n. 2, p. 18-35, maio/ago. 2017. 
El desarrollo de innovaciones en los servicios es valorado como nulo (1), por la gran mayoría de organizaciones encuestadas, entre tanto la opción 4 (alto) representó la opinión de la muestra con respecto al desarrollo de innovaciones en la infraestructura de prestación de servicios a partir del desarrollo de iniciativas de PML (ver figura 4). Los agentes encuestados manifiestan que desarrollan innovaciones ambientales para el ahorro de recursos en sus instalaciones mediante el uso de tecnologías limpias (Severo et al.,2012), por su parte la ausencia de innovaciones en los servicios impide evidenciar en el contexto estudiado, beneficios asociados a la incorporación de productos/servicios amigables con el medio ambiente Dodic et.al. (2010).

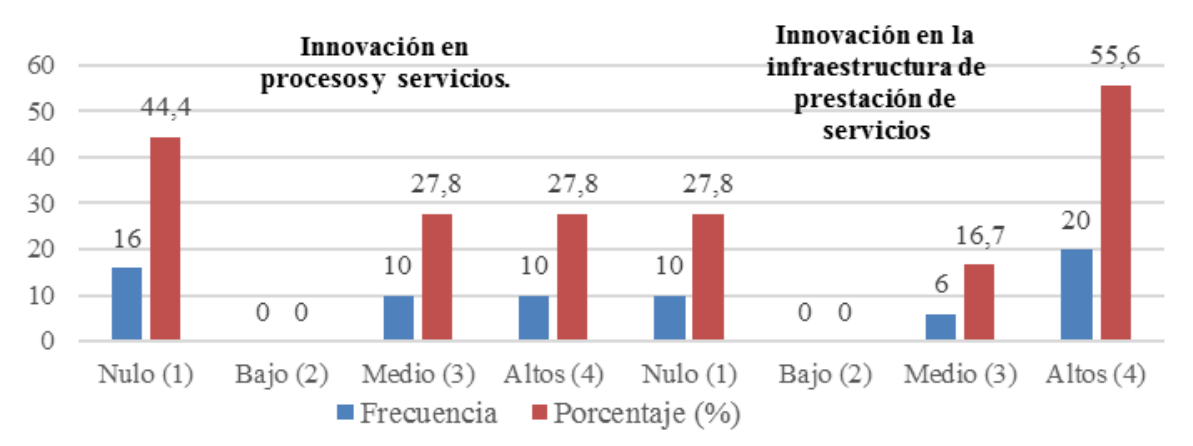

Figura 4. Resultados indicador innovación

Fuente: Elaboración propia (2016) con base en resultados de encuestas (2016)

Los resultados de la valoración realizada por los gerentes con relación a la contribución de las prácticas e iniciativas de RECP al desempeño ambiental y prevención de la contaminación en las organizaciones que representan, se expone en tabla y figura, evidenciándose la presencia de condiciones de efectividad ambiental con un nivel de participación medio, correspondiente a un $60 \%$ y la usual implementación de medidas que permiten controlar o eliminar el consumo de materiales tóxicos en el desarrollo de las operaciones por parte del $94 \%$ de la muestra estudiada, situación que confirma la capacidad de la RECP como estrategia para reducir el impacto en el medio ambiente y la contaminación, mediante un aumento de la eficiencia en el uso de los recursos y la sustitución de materiales peligrosos por insumos que no generen daños a la naturaleza ni a los seres humanos (Organización de las Naciones Unidas para el Medio Ambiente,2003; Molina et al.,2009; Dodic et.al.,2010 y Severo et.al.,2012,2015).

\begin{tabular}{|c|c|c|}
\hline Dimensión & Indicador & $\%$ \\
\hline \multirow{3}{*}{$\begin{array}{l}\text { Efectividad } \\
\text { ambiental }\end{array}$} & $\begin{array}{l}\text { Aumento de la eficiencia en } \\
\text { el uso de los recursos } \\
\text { (Energía-83\%, materiales e } \\
\text { insumos-69\%, agua-69\%) } \\
\text { Moda: } 3 \text { (alto) } \\
\text { Promedio opciones alto (3) } \\
\text { muy alto (4). }\end{array}$ & 74,1 \\
\hline & $\begin{array}{l}\text { Reducción del impacto } \\
\text { ambiental asociado al } \\
\text { consumo del agua. } \\
\text { Moda: } 3 \text { (alto). }\end{array}$ & 53 \\
\hline & $\begin{array}{l}\text { Reducción de la } \\
\text { contaminación. Nivel de } \\
\text { Contaminación generada. } \\
\text { Moda: } 3 \text { (bajo) }\end{array}$ & 53 \\
\hline $\begin{array}{l}\text { Promedio } \\
\text { acumulado. }\end{array}$ & & 60 \\
\hline
\end{tabular}

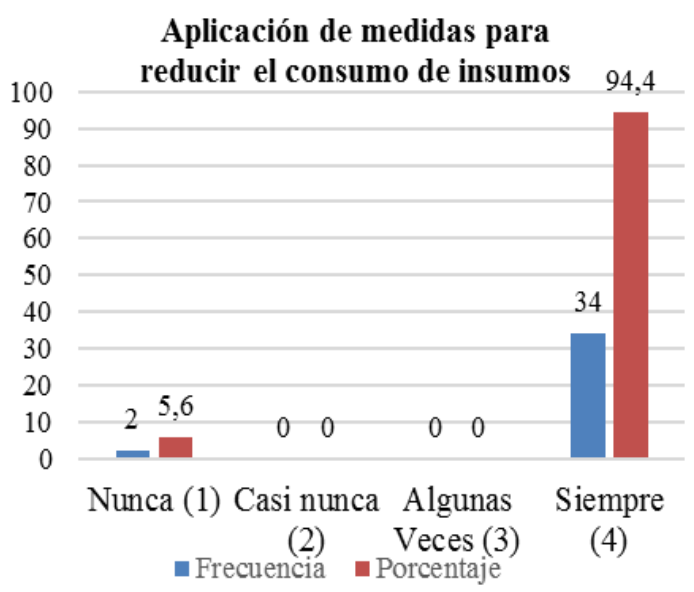

Figura 5. Aplicación de medidas para reducir el consumo de insumos tóxicos.

Fuente: Elaboración propia (2016) con base en resultados de encuestas (2016)

Tabla 6. Resultados dimensión efectividad ambiental.

Fuente: Elaboración propia (2016) con base en resultados de encuestas (2016) 
Los efectos sociales derivados de la implementación de la RECP, son reconocidos en la realidad de las organizaciones objeto de estudio en un nivel medio (69\%), obteniéndose resultados positivos en la reducción de riesgos laborales, la contribución a la calidad de vida de las comunidades y la participación de los grupos de interés en iniciativas de gestión ambiental, tal como se señala en tabla, permitiendo confirmar desde la evidencia empírica y los modelos teóricos propuestos por la Organización de las Naciones Unidas para el Medio Ambiente (2003), Molina et al., (2009), Severo et al, (2012), Severo et al, (2015) y Dodic et.al.(2010) la contribución de la metodología a las condiciones de salud y seguridad en el trabajo, satisfacción de las necesidades de grupos de interés internos y externos, la interacción de los diferentes actores en la implementación de la gestión ambiental, obteniéndose mayor compromiso de las partes y de la organización frente al talento humano, sus condiciones de trabajo y el desarrollo humano en armonía con el medio ambiente.

\begin{tabular}{clc}
\hline \multicolumn{1}{c}{ Dimensión } & \multicolumn{1}{c}{ Indicador } & $\%$ \\
\hline & Reducción de riesgos laborales. & 61,1 \\
\cline { 2 - 3 } Efectividad Social & $\begin{array}{l}\text { Aumento de la Contribución a la calidad de vida de } \\
\text { las comunidades aledañas. }\end{array}$ & 61,1 \\
\cline { 2 - 3 } & $\begin{array}{l}\text { Aumento de la participación de los grupos de } \\
\text { interés. }\end{array}$ & 86,1 \\
\hline & Promedio acumulado. & 69 \\
\hline
\end{tabular}

Tabla 7. Resultados dimensión efectividad social.

Fuente: Elaboración propia (2016) con base en resultados de encuestas (2016)

De igual forma se confirma lo señalado por Henríquez y Catarino (2015), al evidenciarse la reducción de riesgos laborales relacionado con el reemplazo de materias primas toxicas y contaminantes por aquellas menos nocivas para la salud, constituyéndose en otro de los aportes de la aplicación de RECP que genera un impacto directo en la efectividad social alterno a la gestión de variables económicas y ambientales, al permitirles la creación de valor para los clientes internos, cumplir la normatividad vigente en salud y seguridad, mejorar la imagen corporativa, reducir los impactos ambientales negativos de este tipo de sustancias y aumentar la productividad de las personas en la organización.

La participación y contribución de los grupos de interés tanto internos como externos (trabajadores, clientes), desde cada uno de sus roles, es otra de las fortalezas presentes en una alta proporción de las organizaciones estudiadas, comprendida como un beneficio de la RECP según el Programa de las Naciones Unidas para el medio ambiente [PNUMA] (2003), en la medida que propicia la comunicación de los agentes involucrados convirtiéndoles en participantes activos del proceso y de los beneficios derivados de su implementación. Lo antes referido se soporta en una proporción del $95 \%$ de organizaciones que asignan la responsabilidad del ahorro de recursos en toda la organización. La contribución de estos aspectos se manifiesta con una mejora de la imagen, cultura corporativa y la implementación de prácticas de responsabilidad social, lo cual redunda en la competitividad de los entes económicos.

Los resultados obtenidos en cada una de los indicadores y los efectos que un buen desempeño ambiental tiene sobre variables económicas y sociales de los establecimientos permite corroborar lo indicado por el Programa de las Naciones Unidas para el medio ambiente [PNUMA], (2003), Severo et al. (2012,2015), Henriques y Catarino (2014); Dodic et. al, (2010), quienes señalan que la eficiencia en el uso de materiales y energía, las prácticas de carácter ambiental y las metodologías de valor sostenible permiten reducir costos, propiciar comportamientos que garanticen condiciones de higiene, salud y seguridad en el trabajo adecuadas y satisfacer las necesidades o expectativas de los grupos de interés, no obstante en emprender acciones que conduzcan al desarrollo de nuevos servicios (Lin et al.,2016) y la medición de los resultados, se observan debilidades en la muestra estudiada. 


\section{CONSIDERACIONES FINALES}

Caracterizar el aporte real y potencial de la RECP, para la competitividad del sector hotelero del Departamento del Atlántico, permitió comprobar que su aplicación posibilita la obtención de condiciones medias de efectividad económica, ambiental y social en los establecimientos hoteleros y que los beneficios resultantes de un aumento de la eficiencia y reducción de la contaminación se reflejan en el desempeño de variables económicas y sociales, demostrando la complementariedad de la estrategia y su capacidad para articular la gestión ambiental con los aspectos restantes de la gestión organizacional.

La ausencia de un nivel alto de efectividad evidenciable en una tendencia a aplicar actividades de baja inversión, así como limitaciones para desarrollar y materializar innovaciones en procesos y servicios, que contribuyan con la optimización de la producción y prestación de los servicios en la cadena de valor hotelera, impiden aprovechar al máximo el potencial de la RECP para contribuir a la competitividad y están relacionados tal como lo señala Van Hoof et.al (2008) con una perspectiva limitada de sus beneficios e implementación para el desempeño organizacional, que atribuye a las iniciativas de gestión ambiental preventiva y responsabilidad social empresarial una connotación de obligación y gasto carente de todo sentido estratégico (Porter \& Van Der Linde 1995; Porter \& Kramer 2006, Porter \& Krame 2011 a); da lugar al traslado de su valor económico hacia grupos de interés sin encontrar retornos atractivos y genera la necesidad de cambiar los paradigmas de creación de valor económico al uso de metodologías, estrategias e iniciativas que permitan crear valor sostenible (Henriques \& Catarino, 2015).

Los resultados obtenidos y la revisión de la literatura relacionada con las variables, permiten concluir que la RECP, entendida como una estrategia sistémica orientada hacia la generación de valor, contribuye al propósito de trabajar bajo un paradigma de creación de valor compartido, al lograr nuevas formas de operar teniendo como fuente y meta la satisfacción de las necesidades en un proceso de construcción conjunta de métodos, procesos y servicios donde clientes y sectores relacionados participen de los retos, las soluciones y los beneficios que se deriven, convirtiéndose en oportunidades para la empresa en términos de productividad, innovación, satisfacción de necesidades y problemáticas reales de la sociedad

La necesidad de representar gráficamente la transición de la creación de valor económico a la creación de valor compartido y el rol de la $R E C P$ en este proceso a la luz de los resultados obtenidos y los hallazgos de otras investigaciones, dio lugar al desarrollo de un modelo conceptual (ver figura 2 que pretende convertirse en aporte de la investigación al desempeño competitivo, social y ambiental del sector hotelero, asi como punto de partida para otros estudios sobre el tema, al proponer la $R E C P$ como estrategia que al propiciar el desarrollo de prácticas, inversiones e innovaciones genera condiciones de efectividad económica, social y ambiental, con las cuales se integran las tres dimensiones del desarrollo sostenible a la gestión, convirtiendo los factores del entorno en la base para rediseñar servicios y repensar la productividad de su cadena de valor. 


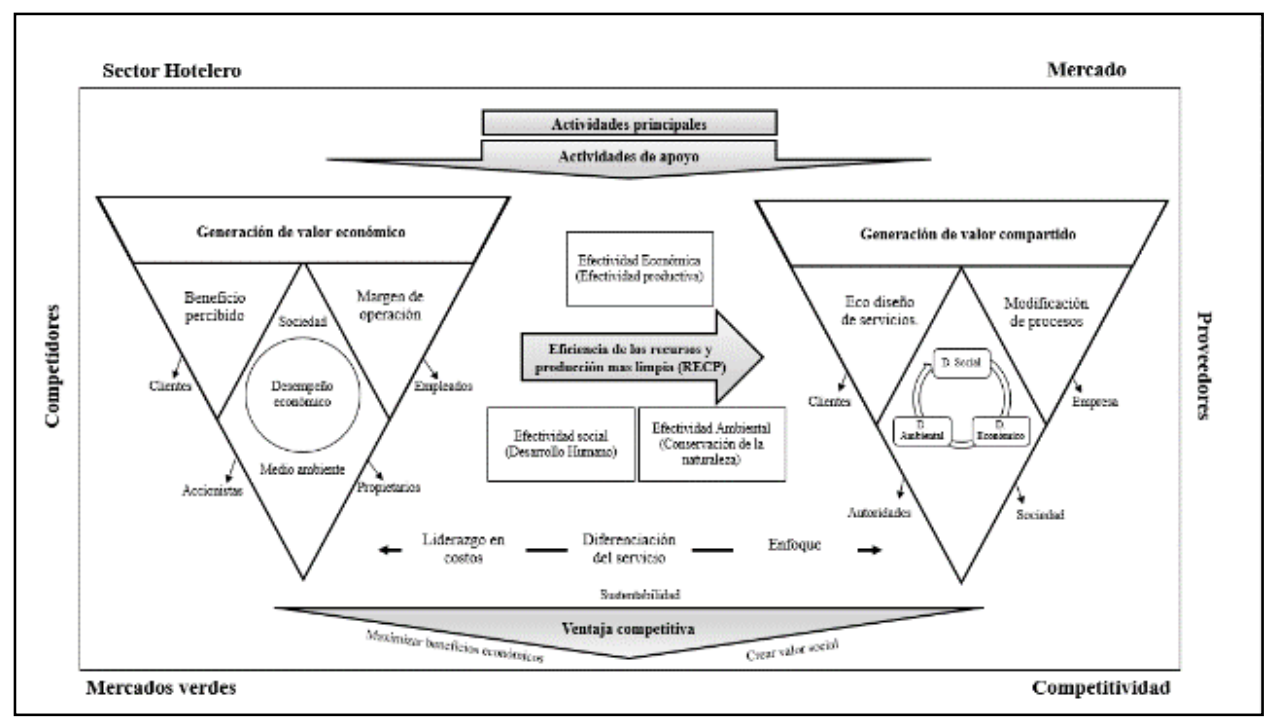

Figura 6. RECP: Enlace Entre La Generación De Valor Económico Y Compartido

Fuente: Elaboración propia (2016), con base en Programa de las Naciones Unidas para el Medio Ambiente [PNUMA] (2003), Porter \& Krame (2011), Fernández (2009); Freeman, Herrigues y Kling (2014).

Desde esta perspectiva, el valor de la empresa se extiende a un número más amplio de grupos de interés, lo cual en lugar de significar una limitación para el desempeño económico se convierte en la oportunidad de construir un vínculo estratégico entre la empresa y la sociedad como aspecto en común que se fortalece con la inclusión de nuevos actores del entorno como agentes aliados para la competitividad de las empresas hoteleras en un entorno inmediato con fuerzas, dinámicas y enfoques alineados a la sostenibilidad, configurando ventajas competitivas más perdurables, así como una oferta de servicios más coherente con las necesidades de la sociedad.

De esta forma, la investigación realizada contribuye al estudio de la RECP desde sus principales y potenciales aportes al desarrollo de estrategias competitivas tradicionales y de creación de valor compartido, como nuevo paradigma para la generación de ventajas competitivas y condiciones de sostenibilidad empresarial

Como limitación del estudio se identifica el tamaño de la muestra analizada, por lo tanto se sugiere para futuras investigaciones cuantificar los beneficios de la RECP para el desempeño competitivo en mayor número de organizaciones contrastándolo con los resultados de las percepciones de los gerentes arrojados en la presente investigación.

\section{Notas}

Artículo científico derivado de la investigación "Producción más limpia como estrategia de competitividad para el sector hotelero del Departamento del Atlántico", financiada por Colciencias y la Universidad de la Costa CUC, adscrita a la línea de investigación Dirección de Organizaciones.

\section{REFERENCIAS}

Alcaldìa Mayor De Bogotà;Unidad De Asistencia Técnica y Ambiental Para la Pequeña y Mediana Empresa [ACERCAR]; Banco Interamericano De Desarrollo [BID]; Asociacion Colombiana De Las Micro, Pequeñas Y Medianas Empresas [ACOPI]; CINSET. (2004). Oportunidades de producciòn màs limpia en el sector hotelero y servicios de restaurante. Bogotà, Departamento Administrativo de medio ambiente (DAMA). 
Alvarez, M., Burgos, J., \& Cespedes, J. (2001). An analysis of environmental management, organizational context and performance of Spanish hotels. Omega The international Journal of Management Science, 29(6), 457-471.

Amado dos Santos, R., Picinini, M., \& Jasmim, M. (2017). Sustainability and hotel business: criteria for holistic, integrated and participative development. Journal of cleaner production, 142, 217-224.

Assenova, M., Georgiev, Z., \& Dunjic, B. (2016). Application of Resource Efficient and Cleaner Production Approach in the Accommodation Sector of the Balkan Region. European Journal of Sustainable Development,5(4), 431-442.

Bernal, C. (2010). Metodología de la investigación: Administración, economía, humanidades y ciencias sociales (Tercera ed.). Colombia: Pearson Educación.

Cabello, J., Sagastume, A., García, D., Cogollos, J., Hens, L., \& Vandecasteele, C. (2015). Bridging universities and industry through cleaner production activities. Experiences from the Cleaner Production Center at the University of Cienfuegos, Cuba. Journal of cleaner production, 108, 873882.

Cabello, J., Sousa, V., Sagastume, A., Alvarez, M., Haeseldockx, D., \& Vandecasteele, C. (2016): Tools to improve forecasting and control of the electricity consumption in hotels. Journal of cleaner production, 137(20), 803-812.

Carbo, D. (2013) Impacto ambiental del sector hotelero en clima calido-humedo. Evaluaciòn y propuestas de mejora. Tesis de maestria,Universidad Politecnica de Catalunya, Barcelona, España.

Cardoso, G., Vendrametto, O., Alencar, I., Palmeri, N., \& Cezar, W. (2016) Environmental impact reduction as a result of cleaner production inmplementation: a case study in the truck industry. Journal of Cleaner Production, 129(25),681-692, julio.

Dodic, S., Vucurovic, D., Popov, S., Dodic, J., \& Zavargo, Z. (2010). Concept of cleaner production in Vojvodina. Renewable and Sustainable Energy Reviews, 14, 1629-1634.

Fernandez, R. (2009). Responsabilidad social corporativa. España: Editorial Club Universitario.

Figge, F., \& Hahn, T. (2004).Sustainable Value added measuring corporate contributions to sustainability beyond eco-efficiency. Ecological Economics,48 (15), 173-187, abril.

Freeman, A., Herrigues, J., \& Kling, C. (2014). The Measurement of Environmental and resource value. Nueva York: Taylor \& Francis.

Glavic, P., \& Lukman, R. (2007). Review of sustainability terms and their definitions. Journal of cleaner production, 15(18),1875-1885, abril.

Gonzalez, J., \& Gonzalez, O. (2005). Perfiles de proactividad Medioambiental: Evidencia en las empresas industriales españolas. Universia Business Review, 5, 92-101, abril.

Henriques, J., y Catarino, J. (2014). Sustainable Value and Cleaner Production e research and application in 19 Portuguese SME. Journal of Cleaner Production, 96(15), 379-386, abril.

Hernandez, R., Fernandez, C., \& Baptista, P. (2014). Metodología de la Investigación. México: Mc Graw Hill Education.

Heskett, J. L. (1997). The service profit chain. New York: The Free Press.

Hurtado, J. (2010). El proyecto de Investigación. Comprensión holítica de la metodología y la investigación. Bogotá: Editorial Quiron.

Instituto Colombiano de Normas Técnicas [ICONTEC], Ministerio de ambiente y desarrollo sostenible. (2006). Norma Técnica Sectorial 5133: Etiquetas ambientales Tipo I . Colombia . 
Instituto de sostenibilidad turistica [IStur]. (Septiembre de 2011). http://hotelessostenibles.com/. Obtenido de http://hotelessostenibles.com/: http://hotelessostenibles.com/que-es-un-hotelsostenible/

Kassinis, G., \& Soteriou, A. (2003). Greening the service profit chain:The impact of environmental management practices. Productions and operations management,16(3), 386-403.

Kjaerheim, G. (2005). Cleaner production and sustainability. Journal of cleaner production, 13, 329339.Li, X., \& Hamblin, D. (2016). Factors impacting on cleaner production: Case studies of Chinese pharmaceutical manufacturers in Tianjin, China.Journal of Cleaner Production,131, 121132, agosto.

Lin, R., Kim, H., \& Yong, G. (2013). Market demand, green product innovation, and firm performance: evidence. Journal of Cleaner Production 40, 101-107.

Luken, Berkel, V., Leuenberger, \& Schwager. (2015). A 20-year retrospective of the National Cleaner Production Centres programme. Journal of cleaner production, 112 , abril,

Martin, F. (2009). Turismo, globalizacion y recursos naturales en América Latina. Privatización y depredación del patrimonio de los pueblos. XXVII Congreso de la Asociación Latinoamericana de Sociología. VIII Jornadas de Sociología de la Universidad de Buenos Aires. Asociación Latinoamericana de Sociología, Buenos Aires.

Mason, P. (2003). Tourism Impacts, Planning and Management. Oxford: Elsevier.

Molina, A., Claver, E., Pereira, J., \& Tarí, J. (2009). Environmental practices and firm performance: an empirical analysis in the Spanish hotel industry. Journal of Cleaner Production, 516-524.

Oreja, J., \& Armas, Y. (2012). Environmental performance in the hotel sector: the case of the Western Canary. Journal of cleaner production, 29(30), 64-72.

Porter, M. (1991). Ventaja competitiva. Buenos Aires: Rei Argentina S.A.

Porter, M., \& Krame, M. (2011). La creaciòn de valor compartida. Harward Business Review, 1-18. Porter, M., \& Kramer, M. (2006). Estrategia y Sociedad . Harvard Business Review, 2-15.

Porter, M., \& Van Der Linde, C. (1995). Green and Competitive:ending the stalemate. Harvard Business Review, 120-134.

Programa de las Naciones Unidas para el Medio Ambiente [PNUMA]. (Diciembre de 2003). Usando producción más limpia para facilitar la implementación de los acuerdos ambientales multilaterales.

Registro Unico Empresarial y social Camaras de Comercio [RUES]- Registro Nacional de Turismo. (2015).

Sanchez, J., \& Cebrián, F. (2015). Turismo de naturaleza en areas protegidas de México; una propuesta de conservación, aprovechamiento y desarrollo local en el Nevado De Toluca. Cuadernos de Turismo. 36, 339-365, septiembre.

Severo, E., Dorian, P., Olea, M., Camargo, C., Nodari, M., \& cruz, R. d. (2012). Cleaner production:Cases of the metal-mechanic automotive cluster of Serra Gaucha. African Jorunal of Business Management, 6(37), 10232-10237.

Severo, E., Ferro, J., Henri, E., \& Hermann, C. (2015). Cleaner production, environmental sustainability and organizational performance: an emprical study in the Brazilian Metal-Mechanic industry. Journal of Cleaner Production, 118-125.

Shen, H., \& Hsieh, T. (2011). An environmental performance assessment of the hotel industry using an ecological footprint. Journal of Hospitality Management and Tourism , 1-11. 
Sulzbach, C., Da Silva, A., \& Hein, N. (2015). Responsabilidade Social e Desempenho EconômicoFinanceiro das Empresas Listadas no Índice de Sustentabilidade Empresarial - ISE. Revista de Gestão Social e Ambiental, 9(2), 53-69.

United Nations Environment Program [UNEP]. (29 de 10 de 2016). Resource Efficient and Cleaner Production. Obtenido de http://www.unep.fr/scp/cp/

United Nations Industrial Development Organization [UNIDO]; United Nations Environment Programme [UNEP]. (2009). Joint UNIDO- UNEP Programme on Resource Efficient and cleaner production in developing and trasition countries: Programme Strategy. Recuperado el Noviembre de 2015, de www.unep.org: http://www.unep.org/recp/

United Nations Industrial Development Organization [UNIDO]; United Nations Environment Programme [UNEP]. (2010). Joint UNIDO-UNEP Programme on Resource Efficient and Cleaner Production in Developing and Transition Countries.

Van Berkel, R. Resource efficient and cleaner production (RECP) for better entreprises, cleaner environment and green Economy. Moldova.

Van Hoof, B., \& Herrera, C. (2007). La evolución y el futuro de la producción más limpia en Colombia. Revista de Ingenieria. (26), 101-117.

Van Hoof, B., Monroy, N., \& Saer, A. (2008). Producciòn màs limpia: Paradigma de gestion ambiental. Barranquilla: Alfaomega Colombiana S.A.

Wahala, W., Edirisinghe, L., Peiris, V., \& Kumarasena, N. (2012). Resource efficient cleaner production (RECP) as a potential tool for greening the hospitality industry in Sri Lanka.

International Forestry and Environment Symposium, 163.

World Tourism Organization (UNWTO). (2016). Annual Report 2016. UNWTO.

World Tourism Organization (UNWTO); United Nations Environment Programme. (2008). Climate Change and Tourism- Responding to Global Challenges . Madrid: World Tourism Organization .

Zeng, S., Meng, X., Yin, H., Tam, C., \& Sun, L. (2010). Impact of cleaner production on business performance. Journal of Cleaner Production, 18, 975-983.

Data da submissão: 04/11/2016

Data de aceite: 02/08/2017

Revista de Gestão Social e Ambiental - RGSA, São Paulo, v. 11, n. 2, p. 18-35, maio/ago. 2017. 